\title{
THE RELATIVE INTENSITIES OF METAL AND GAS SPECTRA FROM ELECTRICALLY CONDUCTING GASES. ${ }^{a}$
}

By P. G. Nutting.

From an arc may be obtained a nearly pure spectrum of the electrodes, the spectrum of the surrounding atmosphere under certain conditions scarcely appearing at all. On the other hand, the light from a discharge tube may give a pure spectrum of the atmosphere about the electrodes, but no spectrum of the electrodes themselves. Various kinds of arcs, sparks, and discharge tubes under various conditions may exhibit mixed spectra in all intermediate proportions.

Arc, spark, and low-pressure discharge are but particular cases of a general case in which a gas forms part of an electric circuit otherwise metallic. It is the purpose of this paper to discuss the many conditions that may govern the preponderance of the spectrum of the electrodes over the spectrum of the intervening gas, select the predominant influences and then if possible to map out the conditions necessary for the production of a given pure spectrum with lines of a given character. To obtain coordinate data over the wide range necessary to warrant general conclusions, the spectra of twenty-one different metals were photographed each under a uniform schedule of eighty. one different conditions.

The relative intensity of the spectrum of the electrodes depends primarily, of course, on the relative proportion of its vapor present in the part of the arc, spark, or discharge tube observed. We are

$a$ This paper is the third of a series of four on the general subject of the preponderance of one spectrum over another in a mixture of two spectra. The first of this series dealt with the conditions governing the relative intensities of the spectra of two mixed elementary gases under low-pressure discharge. (The Spectra of Mixed Gases, Bulletin No. 1, pp. 77-81, November, 1904.) The second dealt with the relative intensities of the two different spectra exhibited by the electro-negative elementary gases. (On Secondary Spectra, Bulletin No. 1, pp. 83-93, November, 1904.) The fourth paper of the series is to be on the spectra of alloys. 
concerned first with the conditions governing the amount of electrode metal raporized and then with the laws governing spectral preponderance in mixed gases, namely; (1) increasing the relative amount of a gas present in a mixture of conducting gases increases the relative intensity of its spectrum; (2) other things being equal, in a steady discharge the spectrum of that component of a mixture having greater atomic weight will be brighter; ${ }^{a}$ (3) in a disruptive or forced oscillatory discharge the atomic weight effect is less.

When the internal oscillations are free (instead of being forced) as in the arrangement used by Ramsey, Lilienfeld, and others to obtain the spectrum of argon in that of ordinary air, perhaps a fourth law would be necessary. Konen (Beibl., 1904, p. 1000) has cited the case of mixtures containing oxygen as apparent exceptions to the atomicweight law (2) above. But oxygen, it must be remembered, has only a very faint (visible) anode glow. Hence to make a fair test of the law we must either take the original faintness of the anode glow into account, or else use the cathode-glow spectrum for comparisons. I have always done the latter. In explanation of the exceptional absence of luminosity in the oxygen anode column, I would suggest that there may be the usual amount of radiation in this case, but that it is chiefly in the infra-red region. The anode column is known to be a region of frequent ionization and recombination, ${ }^{b}$ hence we should expect it would be a region of great chemical changes as well. In oxygen, ozone would be rapidly formed and decomposed; hence the radiation from the anode column would consist largely of the strong infra-red ozone bands recently studied by Angstrom. ${ }^{c}$

The metallic or nonmetallic character of the atmosphere surrounding a spark appears to make no difference whatever with the behavior of the spectrum of the electrodes. A spark between aluminum electrodes in an atmosphere of mercury vapor shows identically the same changes on the introduction of capacity, inductance, spark in series, etc., that it exhibits in hydrogen. On the other hand, the spectrum of an iron spark preserves its invariant character as well in mercury vapor as in air, oxygen, or hydrogen.

The vaporization of the electrodes with which we are concerned in the arc, spark and vacuum tube is by no means a mere temperature effect. The amount of vaporization of an electrode appears to bear no simple relation either to vapor pressure or temperature. It does,

\footnotetext{
a The Spectra of Mixed Gases, Astrophys. J. 19, p. 107; Mar., 1904.

$b$ Thomson: Conduction of Electricity Through Gases, p. 486, ๆ 269.

c Arkiv. för "Matematik," Ast. och Fysik 1. pp. 347-353; 1904. Beiblätter, 28, p. 1142; Nov., 1904.
} 
however, vary enormously with the electrical conditions in the conducting gas, and for this reason a brief discussion of the phenomena of gas conduction is here given.

ELECTRICAL CONDUCTION IN THE ARC, SPARK, AND DISCHARGE TUBE.

Our first problem is to find a relation-a sort of generalized Ohm's law-between the current passing through a gas, the drop in potential and certain constants relating to the gas and the metal used as electrodes. This relation involves the solution of a differential equation containing an unknown function. This function I have constructed, but it gives a nonintegrable form to the differential equation. However, the particular case of steady current admits of a graphical solution (due to W. Kaufmann ${ }^{a}$ ) and covers a wide variety of the phenomena with which we are concerned.

Consider a circuit containing an e. m. f. $E_{0}$, an ohmic resistance $R$, inductance $L$, capacity $C$, and a column of gas through which the drop of potential is expressible by the (experimentally determined) function $e(i)$ of the current. Equating energy supplied to energy taken up by the circuit,

$$
E_{0} i=i^{2} R+L i \frac{d i}{d t}+\frac{q}{C} \frac{d q}{d t}+i e(i)
$$

The final term, $i e(i)$, represents the energy used up in the discharge in whatever manner the drop of potential $e$ varies with the current.

As an illustration, consider the energy used by a column of nitrogen $10 \mathrm{~cm}$ long and at $1 \mathrm{~mm}$ pressure, with platinum electrodes carrying 1 milliampere of current. The anode fall of potential is about 20 volts, the fall through the gas about 600 volts ( 60 volts per $\mathrm{cm}$ ), and the cathode fall 260 volts. Hence the total energy used will be about 0.9 watt, of which about a third is used up at the cathode, two-thirds in the gas, and a negligible amount at the anode. If the column were shortened to $5 \mathrm{~cm}$ the energy used at the electrodes would be the same, while in the gas but half the previous amount of energy would be used up, and the total fall of potential would be 580 instead of 880 rolts. In an ordinary illuminating arc carrying a current of 10 amperes, of the total drop in potential of approximately 50 volts, about half is at the electrodes, so that the luminescent gas receives about half the energy supplied. In the spark we may be reasonably certain that the energy losses are distributed in a manner intermediate between

a Ann. d. Phys. 2, pp. 158-178; 1900.

$2214-$ No. $3-05-8$ 
that of the are and the discharge tube mentioned above. In every case it appear's to require a greater expenditure of energy to force a (positive) current from a gas into a metal than from a metal into a gas.

Now, the loss of energy at the electrodes is not within the electrode itself, nor is it in the adjacent gas outside; it is localized right at the surface separating the electrode from the conducting gas, and represents energy used in forcing the current from the metal to the gas or vice rersa. Apparently all the electrode loss of electrical energy reappears as heat energy, about half being given to the gas and half to the electrode. In the spark with capacity, probably a large part of this energy is used up directly in raporizing the surface layer of the metal, the discharge being so sudden that there is no time for the heat to be communicated to the adjacent metal or gas. In the arc a considerable part of this surface heat is conducted back and radiated from the solid electrode.

The electrical energy equation above can only be solved when the function $e(i)$ is known. This function may be constructed from the properties of its derivative in the following manner: since $e(i)$ has in general a single maximum and a single minimum, but no real roots, write for the derivative $d e=a\left(e-e_{1}\right)\left(e-e_{2}\right) d i$, where $e_{1}$ and $e_{2}$ are the ordinates of the maximum and minimum. By integration

$$
e=e_{2}-\frac{e_{1}-e_{2}}{c e^{a i\left(e_{2}-e_{1}\right)}-1},
$$

a function having the general properties of the characteristic curve for a gas, but which does not give a solvable form when substituted in the energy equation.

When the current is steady the energy equation becomes

$$
E_{\mathrm{o}}=i R+e(i),
$$

from which the value of the current may be obtained (Kaufmann, l. c.) graphically, it being the abscissa of the point of intersection of the line $E_{0}-i R$ with $e(i)$. (The abscissa of the point of intersection of $y=\varphi_{1}(x)$ and $y=\varphi_{2}(x)$ is a root of the equation $\varphi_{1}(x)-\varphi_{2}(x)=0$.) Since in a conducting gas but little energy is stored in comparison with what is dissipated, the $e(i)$ in the last equation is the same as that in the general equation above.

Some typical characteristic curves, $e(i)$, are shown in fig. 1 . The curve marked $g$ shows the drop of potential as a function of the current in its general form. It is to be noted that: (1) the current starts with a brush discharge, during which the drop increases with increasing current; (2) there is a maximum drop at which the gas 
breaks down; (3) after the gas breaks down for a time the drop decreases as the current increases; (4) there is a minimum drop at which the drop is independent of the current, (5) followed by a region in which drop and current increase together. This form of curve may be realized in a gas at moderate pressures with the electrodes at a. moderate distance apart. ${ }^{a}$

The curve $a$, fig. 1 , is characteristic of an ordinary arc. The maximum is very high (say 20,000 volts), while the minimum is low, only about 50 volts. The downward sloping part of the curve is the portion usually observed, using but little series resistance and low e. m. f. To start an arc, like any other form of gas conductor, one must either (1)

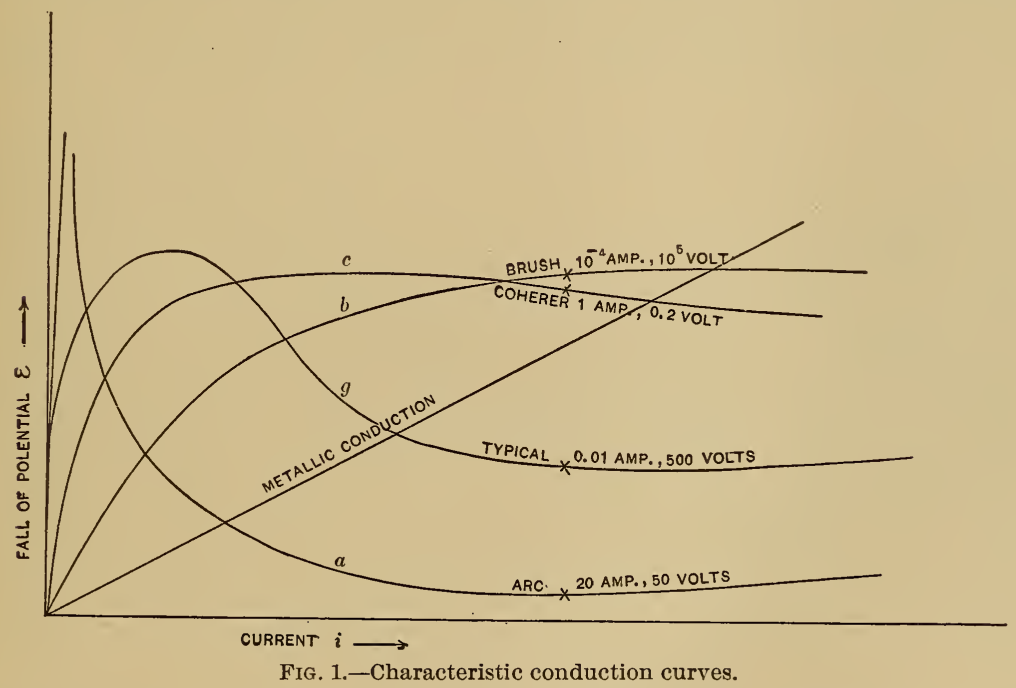

raise the e. $m$. f. above the maximum drop or (2) depress the maximum drop below the e. m. f.; the latter may be done by bringing the electrodes into contact, starting ionization by other means or lowering the pressure on the intervening gas.

The curve $b$ is characteristic of a brush discharge at high pressures between electrodes wide apart or pointed. The electromotive forces concerned are very large, while the currents are small. $c$ is typical of very small distances and high pressures. It was obtained by Guthe and Trowbridge, ${ }^{b}$ using steel balls nearly in contact $\left(10^{-7} \mathrm{~cm}\right.$ apart) as a coherer. The maximum drop is only about one-fourth volt, while

a Studied more in detail, this curve shows steps and other irregularities indicating saturation, ionization by impact, discharge not covering electrodes, etc., but which need not be considered here.

$b$ Phys. Rev., 11, p. 29; 1900. 
the corresponding current is nearly half an ampere. All these curves are seen to be special forms of the type curve $g$, and all are capable of representation by the function $e(i)$ above constructed.

Given the characteristic curve for a gas under given conditions, then by means of the Kaufmann diagram (fig. 2) we may find what the current will be in a circuit containing an e. m. f. $E_{o}$ and an ohmic resistance $R$. The diagram is drawn for a circuit containing a gas at rather low pressure, with a moderately large e. m. f. controlled by considerable resistance. Keeping the e. m. f. $E_{o}$ constant, if the external resistance be increased, the line $E_{0}-i R$ will take a greater and greater slope and intersect the $e(i)$ curve farther and farther to the left. At a certain slope it will intersect the curve in three places and the current may become intermittent. Finally

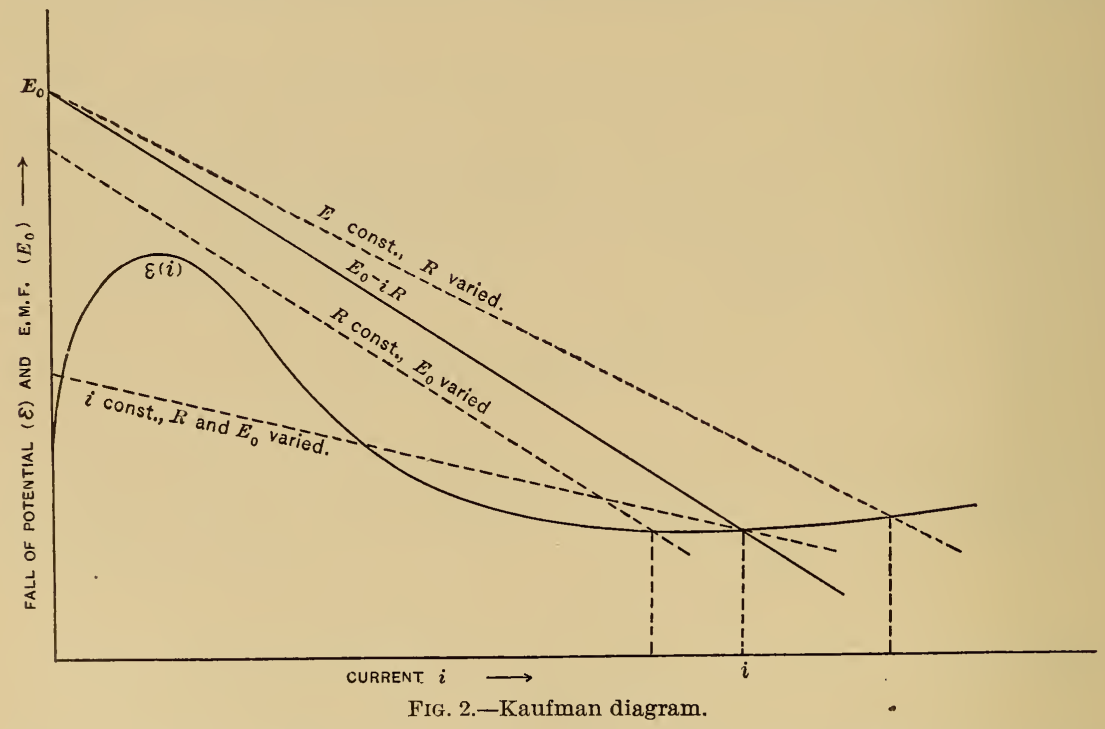

it will become tangent to the under side of the gas curve and the heavy current will cease abruptly, leaving only the brush discharge corresponding to the upper intersection. Diminishing the external resistance, the current will not start again where it ended so abruptly, but only when the resistance line becomes tangent to the curve on its upper side. By taking a higher e. m. f., only single intersections will occur as the resistance is varied, hence the current will never become intermittent and the whole $e(i)$ curve may be followed through experimentally, as it can not be when the e. $\mathrm{m}$. $\mathrm{f}$. used is just sufficient to break down the gas. Stark ${ }^{a}$ has worked out the relation between the critical minimum current for the arc (when the 
arc breaks) and e. m. f. and electrode metal. The relation to the density of the surrounding atmosphere may be seen from figs. 1 and 2, since as the pressure is diminished, curve $a$, fig. 1 , is deformed into curve $g$.

When a spark or Plücker tube is excited by an induction coil or transformer, it is the e. m. f. $E_{o}$ that is variable, while the resistance remains constant. In this case the $E_{o}-i R$ line moves parallel to itself, and the resultant current flowing through the gas has quite a different wave form from what it would have if the current were controlled by varying the resistance alone. Fig. 3, taken from the author's paper on Rectifying Effects, shows a typical curve for the current through a

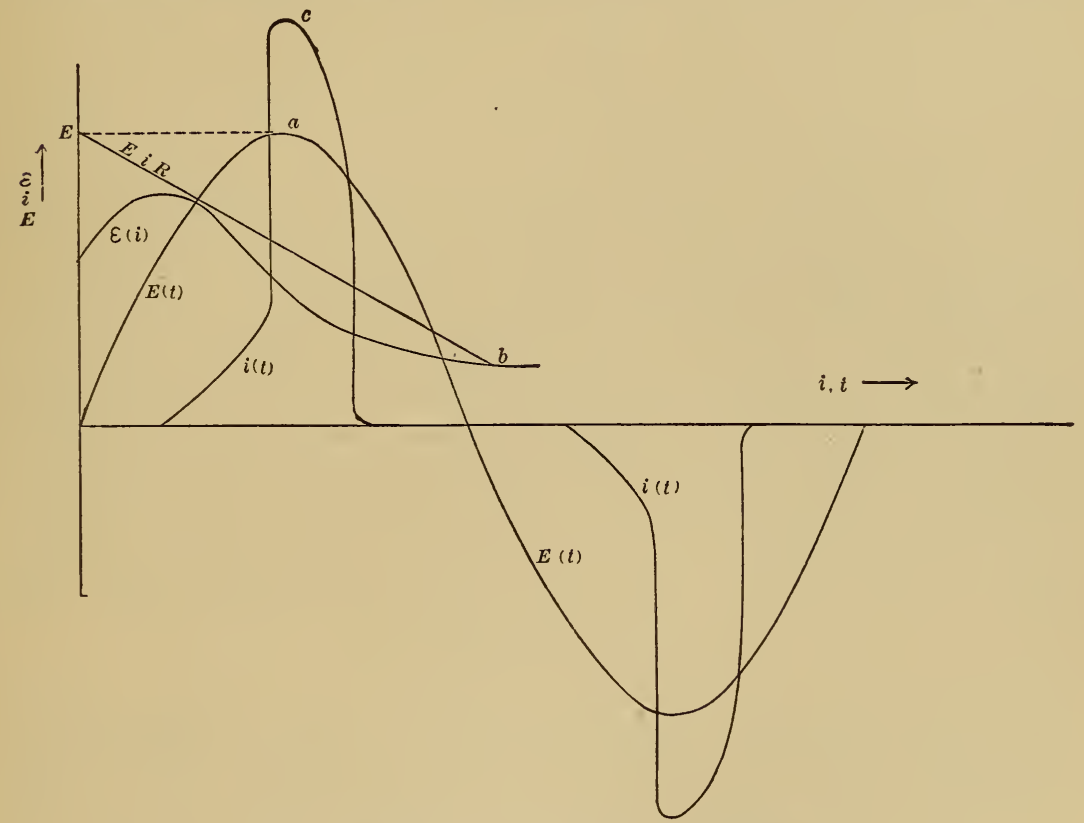

FIG. 3.-Alternating current curve through gases.

gas upon which a sine alternating e. m. f. $E(t)$ is impressed. Starting with the point $a$ on the curve $E(t)$ giving the e. m. f. as a function of time, the point $c$ on the curve $i(t)$ giving current as a function of time is obtained by means of the intermediate points $E$ and $b$, assuming that the characteristic curve $e(i)$ for the gas is of the form shown and that sufficient ohmic resistance has been used to give the line $E-i R$ the slope indicated. Fig. 3 of course represents the current only in the ideal case of a circuit containing neither inductance nor capacity. When inductance $(L)$ is added, instead of $E-i R$ we must use the line $E-i R-L \frac{d i}{d t}$ (a line in three dimensions $E, i, \frac{d i}{d t}$ ) to find the current 
graphically. Without constructing this three dimensional diagram it is easy to see that the general effect of adding inductance is to increase the slope of the $E-i R$ line while the current is starting and to decrease the slope when the current is decreasing. Hence the rertical portions of the current curve $i(t)$, fig. 3 , will be replaced by sloping lines which will always be convex toward the axis of abscissæ and sharp corners will all be rounded off.

When a low voltage transformer (say 1,000 volts) is used to excite a Plücker tube with but little resistance or inductance in series, the current, if it pass at all beyond a mere brush discharge, will pass with brilliant flashes of light. This sudden and very great increase in the current is what we should expect, since in this case in the Kaufmann diagram the line $E-i R$ is nearly horizontal and after it has once passed over the crest of the $e(i)$ curve, intersects the latter only at a very large value of the current.

But it is only after capacity has been added that sufficient quantity of electricity can be supplied to actually attain the enormous currents predicted by the Kaufmann diagram for circuits of low resistance and inductance. The spark with capacity runs through the characteristic $e(i)$ curve for a gas up to large values of $i$; without capacity, an intermittent or alternating current runs over the small current end of the curve only. When the spark passes several times during each alternation, all discharges after the first are made over a modified and much lower characteristic curve. The effect of capacity on the form of the current curve, $i(t)$, fig. 3 , is the opposite of the inductance effect, it sharpens the corners and increases the discontinuities. But quantity not being independent of the current, like $\frac{d i}{d t}$, we can not extend the Kaufmann diagram to cover the case of capacity as was possible with inductance, nor do the equations appear to admit of other than a graphical solution. Aside from making a very heavy maximum current possible, adding capacity has another very important effect generally overlooked, namely, that of reducing the potential of the discharge, or more strictly speaking the maximum e. m. f. of the spark circuit. If the supply of energy is limited, as it is in an induction coil or small transformer, the voltage may be cut down to 10 or 20 per cent of its value when no capacity is in circuit, and cutting down the voltage nearly to that necessary to just cause a spark to pass, increases the discontinuities in the current curve, and hence may actually strengthen the metallic spectrum. I have succeeded in showing this in a very striking manner. A 2 -mm spark in hydrogen at about 
atmospheric pressure was produced by a 300 -watt 5,000-volt transformer controlled by noninductive resistance in its 100-volt side. With only 50 watts supplied, capacity was introduced until the spark barely passed, and thus a brilliant metallic spectrum, sharing the spectral energy about equally with the hydrogen, was obtained. But when the energy supply was increased from 50 to 200 watts the metallic spectrum was weakened to less than one-fourth of the total energy. Introducing inductance did not appear to alter this voltage effect.

Another aspect of the effect of capacity on the spark should be mentioned here. If a spark with large capacity in parallel and fed with a steady supply of current is examined with a rotating mirror, bright sparks appear at regular intervals. On decreasing the capacity gradually the sparks appear smaller and nearer together, until at a capacity of a few centimeters they are no longer distinguishable. The spark has now become an arc, and from this point of view an arc may be regarded as a rapid succession of very minute sparks.

Hartmann, ${ }^{a}$ working with a magnesium arc carrying a current of 4 amperes, found that the line $M g 4481$ appeared much brighter with only 20 volts e. $\mathrm{m}$. f. than it did on a 120 -volt circuit. A Kaufmann dia gram (see fig. 2) indicates a very plausible explanation. The 20 -volt current is just on the point of breaking, hence the $E-i R$ line will cut the characteristic gas curve in three points, while the 120 -volt line cuts it but once. The 20-volt arc should then be of the nature of a rapid succession of very short sparks, and might well show spark lines in its spectrum.

\section{EXPERIMENTAL RESULTS.}

Experimental work was undertaken chiefly with the object of providing a wide range of homogeneous data relating to the conditions governing the relative intensity of the spectrum of the electrodes to the spectrum of the intervening gas in arc, spark, and discharge tube. Spectra of 21 different metals were photographed with a large spectrograph, all under a uniform schedule of 81 different conditions. Three different gases were used as atmospheres; pure hydrogen, pure oxygen, and air, each being used at three different pressures, namely, $760 \mathrm{~mm}, 380 \mathrm{~mm}$, and $100 \mathrm{~mm}$, a considerable number of metals being tried at $4 \mathrm{~mm}$ as well. On each plate nine spectra were taken with the same metal in the same gas at the same pressure, but with nine different modes of excitation, thus making 81 different spectra of each metal. 
The spectra of the following metals were examined:

$\begin{array}{lll}\text { Silver. } & \text { Chromium. } & \text { Palladium. } \\ \text { Aluminium. } & \text { Copper. } & \text { Platinum. } \\ \text { Gold. } & \text { Iron. } & \text { Antimony. } \\ \text { Bismuth. } & \text { Mercury. } & \text { Silicon. } \\ \text { Carbon. } & \text { Magnesium. } & \text { Tin. } \\ \text { Cadmium. } & \text { Nickel. } & \text { Tellurium. } \\ \text { Cobalt. } & \text { Lead. } & \text { Zinc. }\end{array}$

The excitation schedule was as follows:

1. Large capacity-about $0.04 \mathrm{mf}$.

2. Moderate capacity-about $0.02 \mathrm{mf}$.

3. Small capacity-about $0.006 \mathrm{mf}$.

4. No capacity; i. e., 5,000-volt, a. c. arc.

5. Series spark with small capacity-0.006 $\mathrm{mf}$-over both sparks.

6. Same as (1), but with large inductance added-0.9 millihenry.

7. Same as (2), with same inductance as (6).

8. Same as (3), with same inductance as (6).

9. Same as (5), with same inductance as (6).

In the last four cases capacity was varied rather than inductance, because the inductance effect is sharply marked and persistent, while the capacity effects are more varied. These nine spectra, each about $15 \mathrm{~cm}$ long and $4 \mathrm{~mm}$ wide, were photographed side by side on the same plate, so that very slight variations could easily be traced.

A spark about $2 \mathrm{~mm}$ in length was used. This was excited usually by a 5,000 -volt, 300 watt transformer, but sometimes a 5,000 -volt continuous current from a generator set was used instead. The condenser consisted of large glass plates in air and had a capacity of about 0.15 microfarad, variable in steps of $0.006 \mathrm{mf}$. The inductance spool had an inductance of about 0.9 millihenry, variable in 0.009 millihenry steps. The series spark used was between $15 \mathrm{~mm}$ brass balls in air and was lengthened out to nearly its maximum before an exposure was made. The tube for holding the electrodes and inclosing the spark is shown in fig. 4. It was designed so that it could easily be opened for cleaning and interchanging electrodes and yet withstand a powerful and long-continued discharge. Electrodes $E, 2 \mathrm{~mm}$ in diameter and $20 \mathrm{~mm}$ long, were slipped into a roll of platinum foil $P$, which was inclosed in a small glass tube sealed off at $S$ except for a wire leading to the outside. The ground joint $J$ permitted easy removal of the whole. The design prevented conduction of heat to the joint to such an extent that stopcock grease could be used (this contained 1 part rubber, 1 part vaseline, $\frac{1}{3}$ part paraffin). A bulb $B$ proved useful at low pressures as an auxiliary gas reservoir in keeping the pressure constant. A quartz window $W, 40 \mathrm{~mm}$ in diameter and $40 \mathrm{~mm}$ away from the spark, was attached with a hard grade of fusible cement. 
The whole makes a very serviceable spark tube. Gases were prepared electrolytically from a solution of potassium hydroxide and dried with phosphorus pentoxide. At each filling, the tube was flushed several times with the gas while a strong discharge was passing.

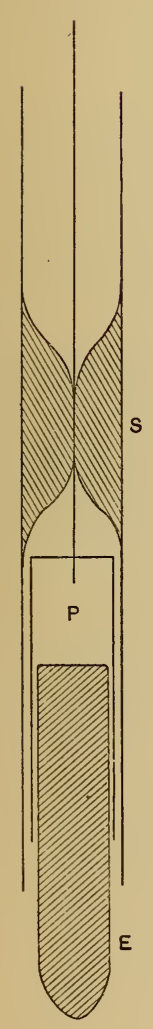

ELECTRODE HOLDER

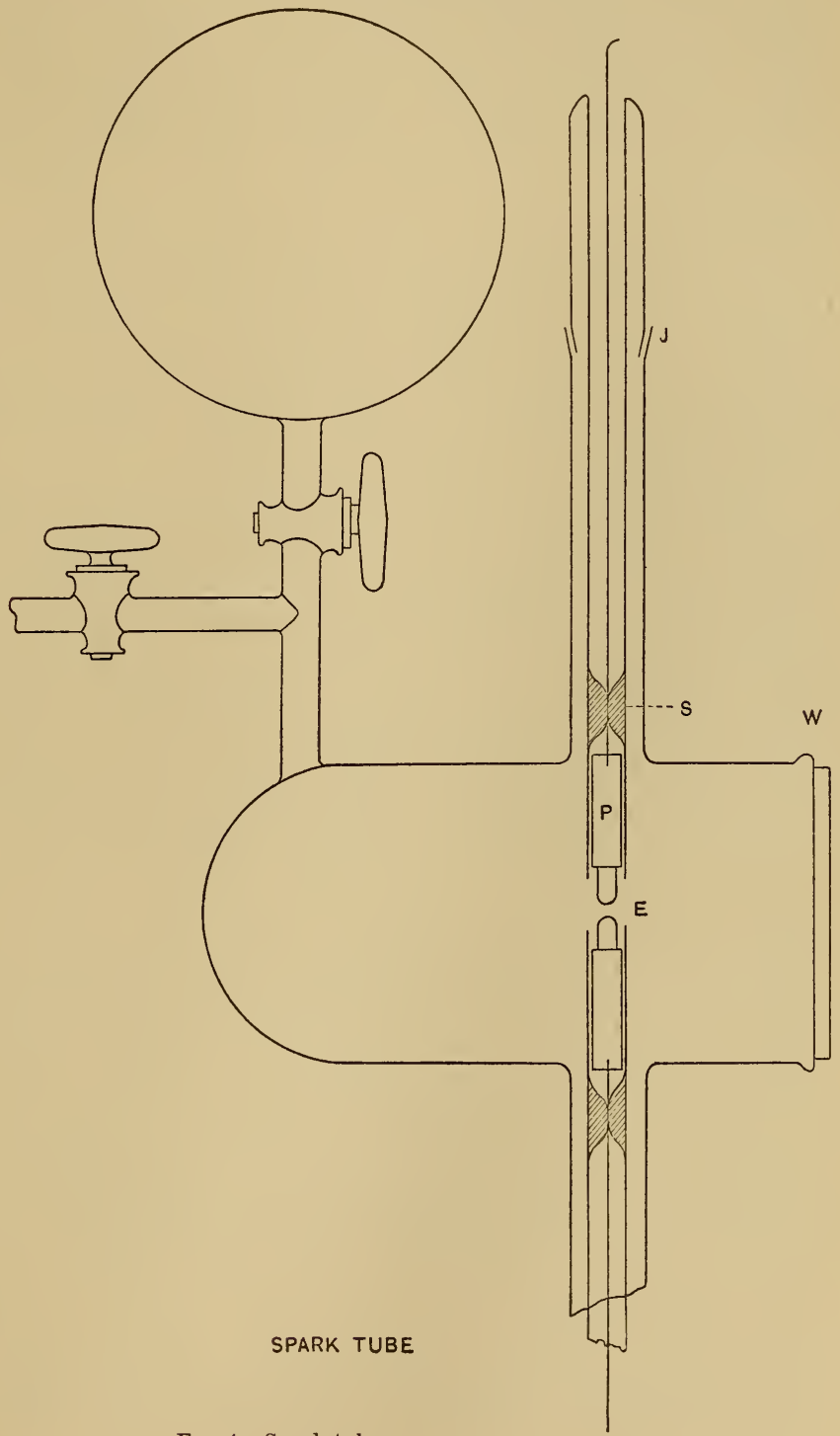

Fig. 4.-Spark tube.

The spectrograms gave of course an integrated effect so far as time was concerned, but effects in different parts of the spark were kept 
separate by throwing a real image of the spark on the slit. Inductance and capacity effects are frequently very different in different parts of the spark; when not specifically stated, the effects here described relate to the central portion of the spark. Changes in the individual spectra were ignored as far as possible in estimating the relative intensity of gas and electrode spectra. When the gas spectrum changed from primary to secondary, the secondary was compared with the metallic spectrum. When inductance cut out part of a metallic spectrum, the remaining spectrum was compared with the gas spectrum.

Throughout the numerous variations in the excitation, the resistance in the primary (100-volt) side of the transformer was the quantity kept constant, with the object of keeping the amount of energy used as nearly constant (300 watts) as possible. The spark gap was usually lengthened out until the spark just passed in the gas at one atmosphere pressure with $0.05 \mathrm{mf}$ capacity in parallel.

In the data given below the intensity of the metallic spectrum is given as a percentage of the whole spectral energy of the spark, electrode and atmosphere together; 30 indicates that 30 per cent of the spectral energy of the aluminium spark-in hydrogen, say-is in the aluminium spectrum, while 70 per cent is in the hydrogen spectrum. These proportions were roughly estimated from prints of the spectrograms, the whole spectrum between the limits $350 \mu \mu$ and $650 \mu \mu$ being taken into consideration. While the accuracy attained is not great, it is ample to determine the conditions affecting spectral preponderance, and fully as great as the constancy of other experimental conditions would warrant.

(1) Variation with electrode metal. - Some metals give relatively a very much stronger spectrum than others under the same conditions. The series runs from $\mathrm{Cu} 10, \mathrm{Pt} 15, \mathrm{Ni} 20$ to $\mathrm{Pb} 75, \mathrm{Bi} 80$, Te 80, other metals being rather evenly distributed between. The numbers relate to sparks with large capacity in hydrogen at atmospheric pressure. The series appears to bear no relation to the fusion temperature series, to the atomic weight series, or to the cathode fall series, nor would the extreme infra-red ${ }^{a}$ and ultra-violet ${ }^{b}$ portions of the spectra, if taken into account, alter the order of the series very materially.

(2) Effect of spark atmosphere.-As a rule, the relative intensity of the electrode spectrum does not vary greatly when the intervening gas is changed. Hydrogen, oxygen, air, and in two cases with aluminium and iron, mercury vapor were used. The spark usually 
appears much brighter in oxygen than in hydrogen, particularly when capacity effects are compared, but the metal spectrum is relatively little if any brighter than in hydrogen. With a majority of metals there appears to be a slight increase from hydrogen to oxygen, while air gives an intermediate intensity. Cobalt in hydrogen 30 , in oxygen 55, in air 40 per cent, is typical. In an atmosphere of mercury vapor the spectra of iron and aluminium were considerably weaker than in the lighter gases, as we should expect from the atomic weight law.

(3) Effect of gas pressure.-Changing the pressure on the atmosphere about a spark over a wide range $(2 \mathrm{~mm}$ to $800 \mathrm{~mm}$ ) does not alter the preponderance of electrode spectrum over gas spectrum sufficiently to be perceptible, and this is true for all the 21 metals tested in the various gases used, and whether excited with or without capacity, inductance, or series spark. This is a most remarkable result, and by no means to be expected. In a very few cases (out of the 1,600 ) - palladium in oxygen with capacity, antimony in hydrogen with capacity and inductance, palladium in air with inductance, nickel and zinc in hydrogen, and magnesium in air with neither capacity nor inductanceslightly different percentages were observed at high and low pressures, but the differences were well within the possible errors due to other causes. Comparing the photographs published by Hale and Kent, ${ }^{a}$ of the spectra of an iron spark at high pressures, with my own, it would appear that the independence of pressure held as well at high pressures, but the broadening, reversal, and overlapping of the lines at high pressures make the results uncertain.

(4) Effect of varying capacity.-The effect of varying the capacity in parallel with the spark from $0.04 \mathrm{mf}$-about all that a $2 \mathrm{~mm} 5,000$ volt 300-watt spark will carry-down to $0.006 \mathrm{mf}$ is scarcely perceptible. As the critical capacity-about $0.01 \mathrm{mf}$ at $760 \mathrm{~mm}^{b}$ and wave length $450 \mu \mu$-is passed, the gas spectrum passes over from secondary to primary, but the intensity of both relative to the metallic spectrum is the same. It is only at very much smaller capacitiesprobably less than $10^{-5} \mathrm{mf}$-that a spark goes over into an arc-i. e., that a rotating mirror fails to resolve the discharge into separate pulses.

$a$ Astrophys. J., 17, p. 154; 1903.

$b$ See B. S. Bulletin No. 1 , p. 87 ; for data on critical capacity. The 180 sets of spectra taken for the present article show well the critical capacity effect in its relation to wave length, pressure, and inductance and independence of electrodes, gas, spark length, and voltage; thus adding considerable weight to the conclusions drawn in the preceding paper. 
(5) Effect of a spark in series.-Putting in circuit a spark in air with $0.006 \mathrm{mf}$ capacity about both the auxiliary and observed spark, produced the same effect as simply adding a large capacity-say, 0.04 mf. The series spark has a great practical advantage over large capacity at low pressures-1 $\mathrm{mm}$ and less-in that it will break up a a glow discharge and give a strong metallic spectrum and secondary gas spectrum even at pressures so low that adding capacity has no effect whatever.

(6) Effect of removing all capacity and inductance.-A spark without capacity is of course an arc, in this case it was an arc at about 4,000 volts fed at about 0.05 ampere. It was usually very faint and frequently at low pressure degenerated into a mere glow about the electrodes, particularly in hydrogen. In hydrogen all electrodes, except those of $\mathrm{Bi}, \mathrm{Hg}, \mathrm{Pb}$, and $\mathrm{Pd}$, show a relatively much weaker spectrum than in the capacity spark. But in oxygen and air all metals showed relatively the same spectral intensity without capacity as with capacity. This effect could hardly have been anticipated and has an important bearing on the general theory following.

(7) Inductance effect. - The effect of inductance in cutting out the air lines from a spark spectrum has been studied before by several workers, but from the results of my own work, I am inclined to question the existence of any such effect, at any rate in atmospheres of hydrogen, nitrogen, or mercury vapor, in the case of any of the twenty-one metals studied. Added inductance does change the secondary spectrum of a gas over into the primary, ${ }^{a}$ in a $2 \mathrm{~mm}$ spark as well as in a $40 \mathrm{~mm}$ Plücker tube, but there is no possibility of anyone familiar with both spectra mistaking the effect. Oxygen lines appear to be swamped by metallic when inductance is added, but what really occurs may be quite different. Oxygen has but an extremely faint primary anode glow spectrum, at least in the visible region. Hence the effect of adding inductance is to change the oxygen spectrum over into an invisible form and not to swamp it with the spectrum of the electrodes. The oxygen lines in the air spectrum being affected in a similar manner, the air spectrum is apparently but not really displaced by the metallic when inductance is added.

(8) Inductance with variable capacity and series spark.-Four of the nine spectrograms on each plate showed the resultant variations of these opposed effects. An inductance of 0.9 millihenry - ten times the critical inductance-was connected in, while capacity was varied from very large to very small and a series spark added. While metallic and gas spectra each show wide variations-various lines appear or dis- 
appear in the metallic spectra while gas spectra change from primary to secondary and vice versa-yet the relative total intensity of metal to gas spectra remains unchanged within an error of 5 to 15 per cent in their estimation. This invariability under such directly opposed conditions is strong confirmation of effects described in paragraphs 4, 5 , and 7. Some very striking differences were observed in the behavior of metallic spectra in oxygen and hydrogen, but they are foreign to the subject of this paper.

An abridged table of results is given below. High, low, and mod-

Table I.-Ratio of Metallic Energy to the Total Energy of Spark.

\begin{tabular}{|c|c|c|c|c|c|c|c|c|c|}
\hline \multirow{2}{*}{ Element. } & \multicolumn{3}{|c|}{$\begin{array}{l}\text { Large capacity and } \\
\text { auxiliary spark. }\end{array}$} & \multicolumn{3}{|c|}{$\begin{array}{l}\text { Inductance with } \\
0.01 \mathrm{mf} \text { capacity. }\end{array}$} & \multicolumn{3}{|c|}{$\begin{array}{l}\text { Arc at } 4,000 \text { volts, } \\
0.05 \text { ampere. }\end{array}$} \\
\hline & $H$ & 0 & Air & $H$ & 0 & Air & $H$ & 0 & Air \\
\hline $\mathrm{Ag}$. & 40 & 20 & 30 & 30 & 90 & 80 & 10 & 20 & 60 \\
\hline $\mathrm{Al}$. & 30 & 50 & 35 & 35 & 50 & 25 & 10 & 40 & 10 \\
\hline $\mathrm{Au} .$. & 35 & 40 & 40 & 35 & 70 & 60 & 30 & 30 & 50 \\
\hline $\mathrm{Bi}$. & 80 & 80 & 70 & 80 & 90 & 90 & 70 & 90 & 70 \\
\hline C ... & 15 & 5 & 10 & 10 & 5 & 10 & 10 & 10 & 10 \\
\hline $\mathrm{Cd} .$. & 45 & 45 & 55 & 70 & 90 & 90 & 20 & 70 & 90 \\
\hline Co... & 30 & 55 & 40 & 40 & 90 & 90 & 40 & 30 & 30 \\
\hline $\mathrm{Cr}$. & 40 & 75 & $\ldots$ & 50 & 95 & $\ldots .$. & 20 & 80 & ...... \\
\hline $\mathrm{Cu} .$. & 10 & 30 & 15 & 10 & 90 & 20 & 10 & 70 & 10 \\
\hline $\mathrm{Fe} .$. & 25 & 70 & 75 & 10 & 90 & 90 & 10 & 60 & $\ldots$. \\
\hline $\mathrm{Hg} .$. & 75 & $\ldots$. & $\ldots$. & 90 & $\ldots$. & ... & 70 & $\ldots$ & ...... \\
\hline Mg. . & 60 & 65 & 50 & 60 & 90 & 90 & 60 & 80 & 30 \\
\hline $\mathrm{Ni} .$. & 20 & 50 & 40 & 15 & 90 & 50 & 10 & 50 & 30 \\
\hline $\mathrm{Pb} \ldots$ & 75 & 80 & 70 & 90 & 95 & 85 & 80 & 80 & 50 \\
\hline $\mathrm{Pd} . .$. & 40 & 45 & 65 & 55 & 85 & 80 & 40 & 70 & 25 \\
\hline Pt ... & 15 & 20 & 10 & 10 & 80 & 40 & 10 & 25 & 10 \\
\hline $\mathrm{Sb} \ldots$ & 35 & 30 & 35 & 65 & 70 & 40 & 10 & 45 & 20 \\
\hline Si... & 35 & 30 & 30 & 30 & 70 & 40 & 10 & 55 & 15 \\
\hline Sn ... & 45 & 30 & 30 & 35 & 95 & 80 & 15 & 65 & 15 \\
\hline $\mathrm{Te} . .$. & 80 & 75 & 30 & 90 & 90 & 70 & 30 & 20 & 25 \\
\hline $\mathrm{Zn} \ldots . . . . . .$. & 30 & 30 & 30 & 40 & 85 & 40 & 10 & 30 & 20 \\
\hline
\end{tabular}

erate pressures are grouped together, as are the effects of large capacity and series spark. The inductance effects quoted relate to 0.9 millihenry used with 0.01 microfarad capacity. The group headed "are" 
relates to sparks without other capacity than lead wires a meter long. Results for carbon (Acheson graphite) are very uncertain on account of the lack of intensity of the carbon spectrum in comparison with compound spectra and the spectra of the impurities (calcium and titanium) present. These foreign spectra were left out of account in the estimate of the ratio of carbon to gas spectra. Values for oxygen with inductance are given as they appeared with no account taken of the shift to infra-red. The mercury spark was about $3 \mathrm{~mm}$ long from mercury to mercury through a hole in the side of an auxiliary glass tube.

(9) Effect of current density. - Varying the current has a pronounced effect in intensifying the spectra of the electrodes at the relative expense of the spectrum of the intervening gas. The effect is well known and considerable data is available. Varying the current varies the amount of vaporization and ionization, and these factors chiefly govern spectral predominance. Vaporization is, of course, not so much due to a heating of the electrode as a whole as to a skin effect due to the great energy lost in the electrode fall of potential at the surface. Whether the electric current first causes a local heating and this heating vaporizes the metal, or whether the current tears the particles directly from the surface and this electrical vaporization causes the heating afterwards we are unable to state, but evidence for the latter process appears stronger. This energy loss at the surface of an electrode being equal to the product of current and electrode fall, the latter being roughly proportional to the current, we should have vaporization approximately proportional to the square of the current. Since the luminosity of a conducting gas is very nearly proportional to the current, the intensity of the spectrum of the spark or arc atmosphere would be proportional to the current. On the other hand the luminosity of the vapor of the electrodes depends on the variable amount of vapor present as well as on the current. Since the amount of the electrodes vaporized is roughly proportional to the square of the current. the luminosity of the electrode vapor would be proportional, approximately, to the cube of the current. Hence a heavy current would greatly assist the preponderance of metallic over gas spectrum.

(10) Effect of electrode temperature.-Raising the temperature of an electrode increases the vapor pressure of the metal, but diminishes the electrode fall of potential, ${ }^{a}$ hence the most advantageous temperature will be an intermediate one, varying widely with the substance used. But in the case of nearly all metals of high melting point both coeffi- 
cients are very small, and the effect of electrode temperature is negligible.

(11) Effect of distance between electrodes.-By varying the length of the column of the conducting gas one may obtain metal and gas spectra in any proportion from pure metal to pure gas. Current, pressure, and distance from an electrode of the observed portion are the chief variable factors to be considered. At high pressures we may have all gradations from an arc to a lightning discharge; at low pressures, everything from an are to a glow discharge. Current and electrode fall of potential determine the amount of metallic vapor that will appear at a given distance from an electrode. Hence at constant current, pressure and distance apart of electrodes need not be considered, except in so far as they affect electrode fall. Since neither anode fall ${ }^{a}$ nor cathode fall ${ }^{b}$ varies greatly with pressure, at constant current the amount of metallic spectrum showing in a given part of a discharge is practically constant. Even in a low-pressure discharge tube $40 \mathrm{~cm}$ long, at a pressure of $0.06 \mathrm{~mm}, \mathrm{I}$ have found the spectrum of the electrodes (aluminum and magnesium) to be a considerable portion of the canal ray glow spectrum.

The effects of varying other conditions than those above discussed appear to be vanishingly small if existent. Varying the e. m. f., for example, has little if any effect so long as current remains constant, account being taken of the very considerable effect on the form of the discharge of varying the control resistance. Nor does the form of the current wave in an intermittent discharge appear to affect the relative intensity of the metallic spectrum. Adding capacity increases the maximum current enormously, but, if the mean current is kept constant, does not appreciably increase the preponderance of the metallic spectrum. The same may be said of the auxiliary spark. Inductance changes only the form of the current wave. Resistance of course would change not only wave form but mean current, but at constant current does not affect metallic preponderance.

\section{Conclusions.}

The results of this investigation show that the same three general laws (p. 400) hold for the distribution of spectral energy between electrodes and gas as hold for other mixed gases. Relative spectral energy depends upon the relative amounts of the two gases or vapors present and, with moderate excitation, upon the atomic weight. The amount 
of electrode vapor present depends upon current and electrode fall of potential, being a surface effect proportional to the energy used in metal-gas conduction. This electrode fall is again a function of a number of other variables. It is nearly proportional to the current, varies slightly with the pressure on the gas and the temperature of the electrodes. The conditions necessary for obtaining a gas spectrum free from the spectrum of the electrodes used, or for obtaining the spectrum of a given metal as free as possible from the spectrum of the surrounding atmosphere, may then be mapped with some certainty. The general case is considered, namely that of a column of gas forming part of an electric circuit that is otherwise metallic.

The conditions favoring a pure gas spectrum are (1) small current density at the electrodes, (2) substance of electrodes to be of some metal like aluminum, copper, or platinum showing small vaporization, (3) electrodes to be at a considerable distance apart and only the middle portions of the discharge are to be used. Gas density, capacity, inductance, series spark, and other conditions have but little effect, and hence may be adapted to other requirements. All three conditions above stated are realized in the Plücker tube, the loss in luminosity caused by the use of small currents being compensated for by the central constriction. In the ordinary arc or spark under the most favorable conditions, it is difficult to obtain a gas spectrum more than 80 per cent pure, whereas by decreasing the current and separating the electrodes, practically 100 per cent purity may easily be realized.

To obtain a pure electrode spectrum, we must have (1) lárge current density and (2) electrodes near together. Oxygen appears to be the most favorable gas to use with the majority of metals, probably on account of the greater cathode and anode fall of potential in this gas. The above conditions are realized in the arc and spark, 95 per cent purity being easily obtainable. Pressure being of little consequence, it may be reduced to give narrow lines, a series spark being added if necessary. Other independent conditions may of course be varied in adaptation to experimental requirements.

March, 1905. 


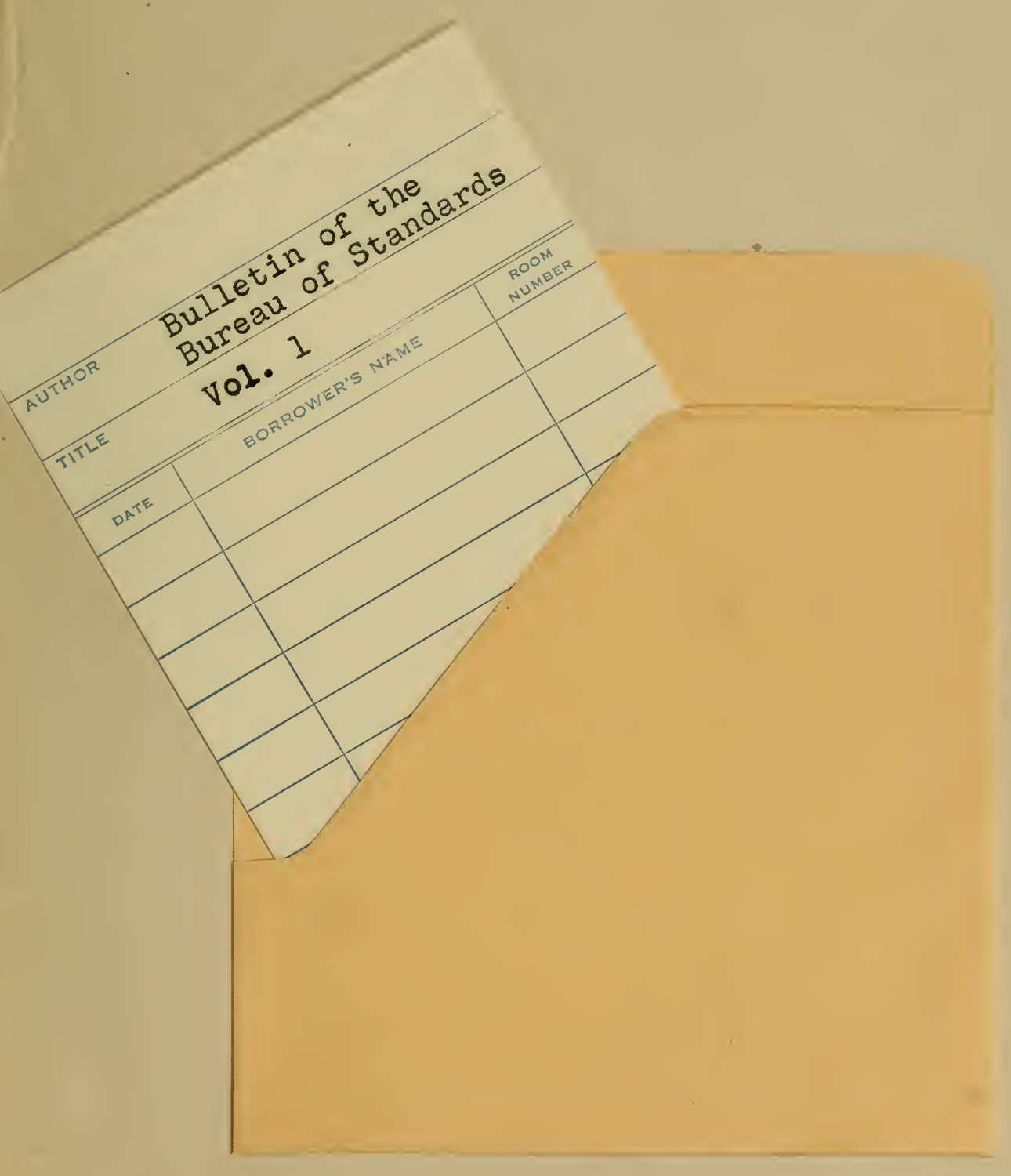


